American Journal of Pediatrics
2019; 5(4): $219-223$
http://www.sciencepublishinggroup.com/j/ajp
doi: 10.11648 /j.ajp.20190504.19
ISSN: $2472-0887$ (Print); ISSN: $2472-0909$ (Online)

\title{
Clinical Profile of Dengue and Predictors of Its Severity Among Children
}

\author{
Sharmin Afroze ${ }^{1, *}$, Salim Shakur², Abrar Wahab ${ }^{3}$, Salomee Shakur ${ }^{4}$ \\ ${ }^{1}$ Department of Neonatology, Dr. M. R Khan Shishu Hospital \& Institute of Child Health, Dhaka, Bangladesh \\ ${ }^{2}$ Department of Paediatrics, United Hospital Limited, Dhaka, Bangladesh \\ ${ }^{3}$ Department of Public Health, North South University, Dhaka, Bangladesh \\ ${ }^{4}$ Department of Medicine, Uttara Adhunic Medical College, Dhaka, Bangladesh
}

\section{Email address:}

mumu.sharmin8@gmail.com·(Sharmin·A.),shakurs@hotmail.com·(Salim·S.), abrar.wahab@northsouth.edu·(Abrar·W.), drshakur.salomee@gmail.com·(Salomee·S.).

${ }^{*}$ Corresponding author

\section{To cite this article:}

Sharmin Afroze, Salim Shakur, Abrar Wahab, Salomee Shakur. Clinical Profile of Dengue and Predictors of Its Severity Among Children. American Journal of Pediatrics. Vol. 5, No. 4, 2019, pp. 219-223. doi: 10.11648/j.ajp.20190504.19

Received: September 26, 2019; Accepted: October 14, 2019; Published: October 23, 2019

\begin{abstract}
Dengue fever is a self-limiting, systemic viral illness that has a wide range of clinical manifestations. Last year dengue was severe in children with much mortality. This study was aimed to see the clinical profile of dengue and to find out the risk factors for developing severity. This cross sectional study was conducted over four months (June-September, 2018) in Department of Pediatrics of United Hospital Limited. All admitted dengue cases were analyzed. The predictors of developing severity were assessed by comparing variables between classical and severe dengue fever groups. A total of 106 children with Dengue fever were admitted. Mean age was $5.8 \pm 3$ years with male predominance $(60 \%)$. Common presentations include fever $(100 \%)$, flushed appearance $(72 \%)$, rash (43\%), vomiting (39\%), abdominal pain (36\%), shock (28\%) and respiratory distress (23\%). In risk factor analysis, overweight (OR: 8.275, CI: 1.378-49.706) and massive serositis (OR: 17.86, CI: 4.733-67.399) were found statistically significant predictors of severe dengue. The overall mortality was $3 \%$. So, overweight children and those patients who develop massive serositis are at increased risk of having severe dengue. Early identification of these features can help physicians to manage these cases judiciously.
\end{abstract}

Keywords: Dengue in Children, Dengue Severity, Risk Factors of Dengue

\section{Introduction}

Dengue is one of the most important arthropod transmitted human viral diseases worldwide including Bangladesh [1]. This virus can lead to significant morbidity and mortality in adults as well as in children. According to World Health Organization, every year 390 million dengue infections occur (95\% credible interval 284-528 million), of which 96 million manifests clinically with any severity of disease [2-3]. A study, conducted in 2012 , estimates that 3.9 billion people in 128 countries are at risk of having dengue [4]. In Bangladesh, the magnitude of dengue fever was largely unknown until it took a heavy toll in 2000 (5555 cases and 93 deaths were reported) $[1,5-6]$. About $90 \%$ of the dengue infections occur in children with risk of dying during a secondary attack is nearly 15 folds higher than that of adults [7]. There are four serotypes of dengue virus which are DENV-1, DENV-2, DENV-3, and DENV-4. Although all four serotypes have similar antigenicity, yet they elicit cross protection for only few months. Secondary infection with another serotype or multiple infections with different serotypes enhance chances of occurring more severe form of diseases [8]. Patients suffering from dengue may be asymptomatic or may present with severe and non-severe clinical manifestations. Common presentations include fever, body ache, rashes etc. But if bleeding and plasma leakage develops, it may be fatal and life threatening. Patients with dengue illness can sometimes develop unusual manifestations such as involvement of liver, kidneys, brain or heart with or without evidence of fluid 
leakage. Such unusual manifestations may be associated with co-infections and co-morbidities and are mostly a result of prolonged shock leading to organ failure. These atypical features if found is defined as Expanded dengue Syndrome [1].

There are many factors which if present in a dengue patient, we can predict severity means possibility of developing dengue hemorrhagic fever with or without shock and even expanded dengue and thereafter manage accordingly. These are obesity, low platelet count, plasma leakage with increasing hematocrit, secondary infection and inadequate fluid management from prior hospitalization etc. [9-12].

Now-a-days dengue fever has become a great public health problem to all tropical and sub-tropical countries in the world as all serotypes /genotypes are now circulating globally and maintaining hyperendemicity. In 2018 Bangladesh experienced an unusual outbreak of Dengue with highest incidence of recorded cases [13-15]. Although children are the main group affected by dengue, little published data are available in Bangladesh. The present study was carried out in order to document the clinical manifestations of dengue infections in children in Bangladesh. We also aimed to identify the risk factors for severity assessment in dengue among children that will further guarantee early identification of risk groups, timely referral and judicious management.

\section{Materials \& Methods}

\subsection{Study Procedure}

This cross-sectional study was conducted from June to September 2018 in department of Pediatrics of United Hospital Limited which is one of the largest tertiary care hospitals in Dhaka. This multi-disciplinary hospital serves a major portion of the North Dhaka community and many complicated cases are always being referred here due to presence of expert professionals along with high quality ICU (intensive care) services. During the study period, all admitted children diagnosed as Dengue (according to Dengue National Guideline 2018) where enrolled in the research work. A written informed consent was taken from parents and a structured questionnaire was used to collect data. Baseline characteristics were recorded. Common clinical and laboratory parameters were also documented. Nutritional status was assessed by using BMI (Body Mass Index) for age percentile according to $\mathrm{CDC}$ [16]. Dengue cases were classified into classical dengue and severe dengue fever group (Dengue hemorrhagic fever and dengue shock syndrome) as per National guideline [1]. In this study, we also analyzed the possibility of overweight $\left(\mathrm{BMI}>90^{\text {th }}\right.$ percentile), massive serositis (gross unilateral pleural effusion or bilateral pleural effusion or moderate to gross ascites or combination of any of these), plasma leakage and low platelet count $(<20,000 / \mathrm{cmm})$ in causation of disease severity. In all admitted patients, the treatment modalities were also observed and outcome was measured as discharge or death.

\subsection{Statistical Analysis}

After collection, data was analyzed using the statistical package for social sciences (SPSS) version 21.0. Quantitative data were expressed as the mean \pm standard deviation and categorical data were presented as frequency. Chi square test was performed to compare qualitative variables. Risk factors for severe dengue were assessed using logistic regression tests to see the odd ratio. P-values $<0.05$ were considered statistically significant.

\section{Results}

\subsection{Baseline Characteristics}

During the study period (dengue season: June to September) a total 106 children with dengue were admitted in United Hospital. The mean age was found $5.8 \pm 3$ years and males were predominant $(60 \%)$. Table 1 show that fifty one percent patients' age was between 5-9 years.

Table 1. Baseline Characteristics of study population

\begin{tabular}{lll}
\hline Variable & Number $(\mathbf{n})$ & Frequency $(\%)$ \\
\hline Age & & \\
Mean age & $5.8 \pm 3$ years & \\
$<$ 1 years & 6 & 6 \\
1-4 years & 32 & 30 \\
5-9 years & 54 & 51 \\
10-14 years & 14 & 13 \\
Sex & & \\
Male & 64 & 60 \\
Female & 42 & 40 \\
\hline
\end{tabular}

\subsection{Clinical Presentation}

During admission, dengue cases presented with variety of clinical features. High grade continued fever was present in $91 \%$ patients and mean duration of fever was $4 \pm 2$ days. Table 2 show that, in $55 \%$ patients, fever lasted for 5 days or more. Along with fever other predominant clinical presentations were flushed appearance $(72 \%)$, characteristic rash $(43 \%)$, vomiting (39\%), abdominal pain (36\%) and headache (33\%).

Table 2. Clinical presentation of study population.

\begin{tabular}{lll}
\hline Variable & Number (n) & Frequency (\%) \\
\hline Fever & 106 & 100 \\
Mean duration of fever & $4 \pm 2$ days & \\
Fever lasting $<5$ days & 24 & 45 \\
Fever lasting $\geq 5$ days & 30 & 55 \\
Flushed appearance & 76 & 72 \\
Characteristic rash & 46 & 43 \\
Cough/ runny nose & 20 & 19 \\
Respiratory distress & 16 & 15 \\
Headache & 35 & 33 \\
Retro- orbital pain & 4 & 3.7 \\
Vomiting & 34 & 39 \\
Abdominal pain & 38 & 36 \\
\hline
\end{tabular}

During classification of Dengue as per National Guideline, 
classical dengue was found more (68\%) and 32\% children had severe dengue in the form of hemorrhage (3\%) and shock $(29 \%)$ in our study (Figure 1).

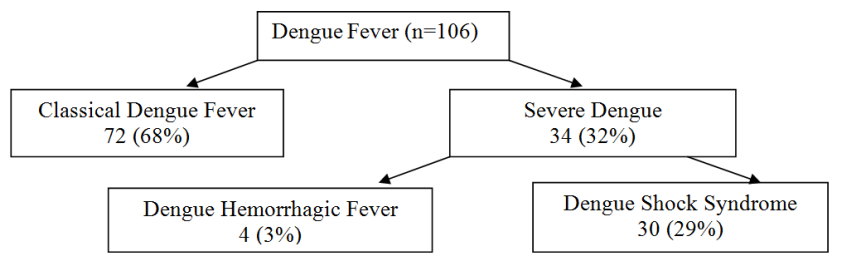

Figure 1. Classification of Dengue cases.

\subsection{Laboratory Parameters}

Table 3. Laboratory Parameters of dengue patients.

\begin{tabular}{lll}
\hline Parameter & Mean & \pm SD \\
\hline Haemoglobin $(\mathrm{gm} / \mathrm{dl})$ & $9.0-18.0$ & 1.5 \\
Hct $(\%)$ & $28-51.8$ & 4.11 \\
Initial WBC $(/ \mathrm{cmm})$ & $1700-17400$ & 2953 \\
Initial Platelet count $(/ \mathrm{cmm})$ & $8400-211000$ & 44022.801 \\
SGPT $(\mathrm{U} / \mathrm{L})$ & $16-183$ & 27 \\
\hline
\end{tabular}

Table 4. Distribution of serologically positive dengue patients.

\begin{tabular}{lll}
\hline Serological test & Patients with Positive & Frequency (\%) \\
\hline NS1 & result (n) & 87 \\
IgM & 92 & 15 \\
Both IgG and IgM & 16 & 11 \\
\hline
\end{tabular}

Baseline laboratory data were observed in the study population (Table 3). Mean Hematocrit level on admission was $28-51.8 \pm 4.11$. Regarding serological tests, Dengue NS1 was found positive (evidence found before or after admission) in $87 \%$ affected children whereas only $15 \%$ had positive result for dengue IgM (Table 4).

\subsection{Severity Assessment for Dengue Fever}

To predict the association of severe dengue with overweight, massive serositis, plasma leakage and low platelet count; these variables were compared between classical dengue fever group and severe dengue fever group (Table 5).

Table 5. Comparison of variables between Severe Dengue and Classical Dengue.

\begin{tabular}{llll}
\hline Variable & $\begin{array}{l}\text { Severe Dengue } \\
\text { fever }(\mathbf{n = 3 4})\end{array}$ & $\begin{array}{l}\text { Classical Dengue } \\
\text { fever }(\mathbf{n = 7 2})\end{array}$ & $\boldsymbol{P}$ value \\
\hline Overweight & 17 & 2 & 0.03 \\
$\begin{array}{l}\text { Massive serositis } \\
\text { Hct increase }>20 \%\end{array}$ & 28 & 6 & 0.00 \\
$\begin{array}{l}\text { Platelet count } \\
<20,000 / \mathrm{cmm}\end{array}$ & 93 & 63 & .485 \\
\hline
\end{tabular}

Statistical test: Chi square test $(* p$ value considered significant when $<0.05$ )

This revealed that children having overweight or massive serositis or low platelet count $(<20,000 / \mathrm{cmm})$ were more at risk of severe dengue $(P$ value $<0.05)$.

In Table 6, a binomial logistic regression was carried out to look for the risk factors of severe dengue. It showed that risk of having severe dengue is 8 folds more among overweight children than normal weight children and we also have found that patients with massive serositis are at 17 folds more risk of getting severe dengue than those who do not have massive serositis $(P$ value $<0.001)$.

Table 6. Logistic regression analysis of risk factors for Severe Dengue.

\begin{tabular}{lllll}
\hline Risk Factor & Reference & $\boldsymbol{\beta}^{*}$ & OR $^{*}$ & 95\% CI \\
\hline Age (1-7 years) & $8-14$ years & -.913 & 0.401 & $0.108-1.487$ \\
Overweight (Yes) & No & 2.113 & 8.275 & $1.378-49.706$ \\
Massive serositis (Yes) & No & 2.883 & 17.86 & 0.579 \\
Platelet $<20,000 / \mathrm{cmm}$ & $\geq 20,000 / \mathrm{cmmm}$ & 0.730 & 2.075 & 0.021 \\
\hline
\end{tabular}

$(* \beta=$ Beta co-efficient, $\mathrm{OR}=$ Odds Ratio, $\mathrm{CI}=$ Confidence interval, $\mathrm{p}$ value significant when $<0.05)$

Regarding management of dengue patients, crystalloid fluid was given in $100 \%$ cases. But $23 \%$ required both colloid and crystalloid fluid. Fresh plasma and platelet transfusion were also used in $28 \%$ and $16 \%$ patients respectively. Frusemide pump was used in 4 patients with good clinical outcome (all survived). During admission $29 \%$ patients were directly admitted into ICU due to presence of warning signs or alarming platelet count or severe dengue manifestations. Among other patients, 12 were transferred to ICU later due to development of complications. Overall mortality from dengue was 3\%. The four death cases were due to Expanded Dengue Syndrome. Among them one case was serologically negative but later diagnosis was confirmed by PCR. Mean hospital stay was $6 \pm 2.5$ days.

\section{Discussion}

In the present study, mean age of children was $5.8 \pm 3$ years and common age group affected was between 5-9 years. Sarker A et al. and Ahmed FU et al. reported that maximum number of cases were below10 years in their work [17-18] which is similar to our study findings. Males were affected more $(60 \%)$ than females in our study which was found consistent with various previous studies [19-21]. In this study $100 \%$ patients presented with fever followed by flushed appearance $(72 \%)$, rash $(43 \%)$, vomiting $(39 \%)$ and abdominal pain (36\%). Similar finding has been found by Alam S et al. and Mobarak MR et al. [5, 19]. But the findings differ from that of Ahmed FU et al. and Rahman M et al. where predominant presentation was headache $(85 \%$ and $91 \%$ respectively) $[18,22]$. In our study headache was found in $33 \%$ and retro-orbital pain was found only in $3.7 \%$ children. As most of the children were below 10 years in this study, so they might not be able to describe these presentations clearly. Regarding laboratory parameters, the mean platelet count of the patients on admission was 
$8400 / \mathrm{mm}^{3}$ which was below $1,00,000 / \mathrm{mm}^{3}$. The mean hematocrit was found $33 \%$ and the mean ALT was $210 \mathrm{U} / \mathrm{L}$. Like Alam S et al. leucopenia was found less in our study that was $11 \%$ of the children [5]. Among the 106 dengue patients, $92(87 \%)$ were NS1 positive, $16(15 \%)$ were IgM antibodies and $12(11 \%)$ were both $\operatorname{IgG}$ and $\operatorname{IgM}$ antibodies positive. This isolated IgM antibody and both IgG and IgM antibodies were found lower than that of Alam S et al.'s study findings (IgM and $\operatorname{IgG}$ antibodies for dengue positive in $40.7 \%$ and $24 \%$ cases respectively and both positive in $31 \%$ cases) [5]. During June to September, when there is peak of dengue, all suspected cases with 1-2 days of fever were advised to do dengue NS1 test. And due to wide availability of this test, probably our study showed high percentage of patients with positive result (Dengue NS1) whereas IgM was positive in less number. The current study also assessed the probability of overweight, massive serositis, plasma leakage and low platelet count as risk factors for severity. These factors have been observed in many other studies separately and significant associations were found [9-12]. In a metaanalysis it was reported that obesity is a risk factor for severe dengue [23]. In obesity there is increased production of white adipose tissue which causes increased production of mediators like IL-1, IL-6 and TNF- $\alpha$. These mediators increase capillary permeability and thus underlie the process of progressive and severe plasma leakage [24]. Based on the same hypothesis, overweight can also lead to severity. So, we tried to find the association and found that overweight children have 8 times increased risk of having severe dengue. We all know that serositis in the form of pleural effusion or ascites can develop in dengue patients as complications due to plasma leakage. But often in clinical practice it is seen that most of dengue patients with massive serositis are more vulnerable to respiratory distress, infections, need of mechanical ventilation and finally fatal outcome. For this there are no studies till date. So, we tried to detect the association and we found that massive serositis can be an early predictor of severity (risk is 17 times for severity). High hematocrit and low platelet count were also addressed in this study in order to get significant association but it was not found. Most of our patients responded well with judicious fluid management and smaller percentage required plasma and platelet transfusion. Ninety seven percent patients were discharged to home which was similar to other study results done in Bangladesh [19]. Overall mortality was 3\%; all of them were severe variety with expanded dengue syndrome.

\section{Conclusion}

The incidence and severity of Dengue in children is increasing every year. Most of the children presents with high grade, continued fever and characteristic rashes followed by vomiting and abdominal pain. Among these patients, those who are overweight and those who develop massive serositis are at increased risk of having severe form of dengue either haemorrhage or shock. So, dengue affected children need strict supervision from admission to hospital stays regarding weight recording, judicious fluid management and monitoring for serositis.

\section{Recommendations}

i. Considering the above-mentioned risk factors, pediatricians should assess nutritional status of dengue affected children on admission by using the BMI chart which can help to identify overweight patients.

ii. In the other hand, daily monitoring of patient's status in the progression of mild serositis to severe form should be addressed. It will help in early detection of the group of children who may deteriorate further. This will also help doctors to treat the cases judiciously and timely referral to ICU with proper counseling.

\section{Conflict of Interests}

The authors report no conflicts of interest. The authors alone are responsible for the content and writing of the article.

\section{Acknowledgements}

All the authors acknowledge United Hospital Limited, Dr. Md. Moshiur Rahman (Senior consultant), Dr. Nargis Ara Begum (Senior consultant), Dr. Ashraf Ali Sheikh (Senior consultant) and the participants for giving permission and providing necessary information to conduct the study.

\section{References}

[1] National Guideline for clinical management of Dengue syndrome. $4^{\text {th }}$ edition, 2018.

[2] WHO fact sheets- Dengue and severe dengue. April 2019.

[3] Bhatt S, Gething PW, Brady OJ, Messina JP, Farlow AW, Moyes CL et.al. The global distribution and burden of dengue. Nature; 496: 504-507.

[4] Brady OJ, Gething PW, Bhatt S, Messina JP, Brownstein JS, Hoen AG et al. Refining the global spatial limits of dengue virus transmission by evidence-based consensus. PLoS Negl Trop Dis. 2012; 6: 1760.

[5] Alam S, Sadat S, Swapan Z, Ahmed A, Karim N, Paul HK, ZamanS. Clinical Profile of Dengue Fever in Children. Bangladesh J Child Health. 2009; 33 (2): 55-58.

[6] Dengue fever and dengue haemorrhagic fever. World Health Organization. 2009.

[7] Prevention and control of dengue and dengue hemorrhagic fever: comprehensive guidelines. World Health Organisation. 1999.

[8] CDC 2007. Center for Disease Control, CDC Health Center. Georgia, USA.

[9] Kalayanarooj S, Nimmannitiya S. Is dengue severity related to nutritional status? Southeast Asian J Trop Med. Public Health. 2005; 36: 378-84. 
[10] Chuansumrit A, Puripokal C, Butthep P, WongtirapornW, Sasanakul W, Tangnararatchakit $\mathrm{K}$, et al. Laboratory predictors of dengue shock syndrome during the febrile stage. Southeast Asian J Trop Med Public Health. 2010; 41: 326-32.

[11] Sutaryo. Dengue. Yogyakarta: MedicaFakultasKedokteran UGM; 2004.

[12] Tantracheewathorn T, Tantracheewathorn S. Risk factors of Dengue shock syndrome in children. J Med Assoc Thai. 2007; 90: $272-7$.

[13] Islam TQ. Changing Epidemiological and Clinical pattern of Dengue inBangladesh 2018. J MEDICINE 2019; 20: 1-3.

[14] Rodhain F, Rosen L. Mosquito vectors and dengue virusvector relationships. In: Gubler DJ, Kuno G, editors. Dengueand Dengue Hemorrhagic Fever. New York: CAB. International; 1997; 45-60.

[15] Gubler DJ. The changing epidemiology of yellow fever anddengue, 1900 to 2003: Full circle? Comp ImmunolMicrobiolInfect Dis 2004; 27: 319-30.

[16] CDC BMI for age growth chart. Centers for Disease Control and Prevention. Factsheet 2018.

[17] Sarker A, Tara PHDAR d, Chatterjee S. Molecular typing of dengue virus circulating in Kolkata India in 2010. Journal of tropical medicine. 2012; 13 .

[18] Ahmed FU, Mahmood BC, Sharma JD, Hoque SM, Zaman R,
Hasan MS. Dengur and Dengue Haemorrhagic fever in Children during the 2000 outbreak in Chittagong, Bangladesh. Dengue Bulletin 2001; 25: 33-39.

[19] Mobarak MR, Islam R, Bhuiya AKM, Akand N, Begum F. Evaluation of Dengue Fever in a tertiary care children hospital of Bangladesh. Northern International Medical College Journal.2017; 9 (1): 274-277.

[20] Gupta E, Dar L, Kapoor G, Broor S the changing epidemiology of dengue in Delhi, India. Virology Journal. 2006; 5 (3): 1.

[21] Chakravarti a, Kumaria R. Eco-epidemiologycal analysis of dengue infection during an outbreak of dengue fever, India. Virology journal.2005; 14 (2): 1.

[22] Rahman M, Rahman K, Siddque AK, Shoma S, Kamal AH, Ali KS, et al. FirstOutbreak of Dengue Hemorrhagic Fever, Bangladesh. Emerg Infect Dis2002; 8: 738-40.

[23] Zulkipli MS, Dahlui M, Jamil NS, Peramalah D, CheeWai HV, Bulgiba A. The association between obesity and dengue severity among pediatric patients: A systematic review and meta- analysis. PlOSNegi Trop Dis. 2018. https://doi.org/10.1371/journal.pntd.0006263

[24] Juffrie M, Meer GM, Haasnoot K, Sutaryo, Veerman AJ, Thijs LG. Inflammatory mediators in dengue virus infection in children: Interleuki -6 and its relation to C-reactive protein and secretory phospholipase A2. Am J Trop Med Hyg. 2001; 65: $70-75$. 